Award (CTSA) site at the University of Michigan, is adopting human-centered design (HCD) to advance the science of community engagement in translational research and CE's programmatic efforts. METHODS/STUDY POPULATION: The MICHR CE Program supports academic-community partnerships to transform translational research across the state of Michigan. As the team aims to better engage partners to help guide the direction of their work, $\mathrm{CE}$ is collaborating with MICHR's Design and Innovation Core to incorporate human-centered design (HCD). HCD is an approach that prioritizes the needs, values, and perspectives of direct users during the creation of a new product or service. The MICHR team created interactive HCD activities for two statewide retreats to elicit feedback from community and academic members on ways to enhance community-engaged research (CEnR). Retreat participants worked on a variety of problems, such as barriers to partnering and defining impact for CEnR. These activities generated authentic, contextual, and multi-view data captured in various artifacts for systematic analysis. RESULTS/ANTICIPATED RESULTS: In the first retreat, a HCD activity had participants reflect on their own barriers to partnering in research and potential solutions. In the second retreat, an HCD activity facilitated participants interviewing each other on their views of the impact in CEnR. Results from the first activity identified a set of common barriers to CEnR, some related to partnership formation, communication, and partner equity, among others. These led the CE Program to specific programmatic efforts, such as designing a statewide partnership platform, hiring a communication program manager, and sponsoring community partners to join national conferences. The second retreat activity produced rich data to identify overlaps between different perspectives to inform how impact can be defined and measured in CEnR. DISCUSSION/ SIGNIFICANCE OF IMPACT: HCD activities provide means to include community and academic members in the science of CEnR. They allow systematic ways to gather information directly from the diverse set of current or prospective partners of community engagement programs about their needs, experiences, and values, which can be translated to programmatic innovation.

4366

Aligning community-engaged research competencies with online training resources across the Clinical and Translational Science Award Consortium

Rebecca Jane Piasecki ${ }^{1}$, Rebecca J Piasecki ${ }^{2}$, Lisa D Quarles ${ }^{3}$, Mona $\mathrm{N}$ Bahouth ${ }^{1}$, Anwesha Nandi ${ }^{3}$, Alicia Bilheimer ${ }^{3}$, Lori CarterEdwards ${ }^{3}$, and Cheryl R Dennison-Himmelfarb ${ }^{2}$

${ }^{1}$ Johns Hopkins University School of Medicine; ${ }^{2}$ Johns Hopkins University School of Nursing; ${ }^{3}$ North Carolina Translational and Clinical Sciences Institute

OBJECTIVES/GOALS: The extent that Clinical and Translational Science Award (CTSA) programs offer resources accessible online for training in community-engaged research (CEnR) core competencies is unknown. This study cataloged CEnR resources accessible online from CTSAs and mapped resources to CEnR core competencies. METHODS/STUDY POPULATION: Eight domains of CEnR core competencies were identified: knowledge/perceptions of CEnR; personal traits necessary for CEnR; knowledge of/relationships with communities; training for performing CEnR; CEnR methods; program evaluation; resource sharing and communication; and dissemination and advocacy. A systematic review of CEnR resources accessible online from CTSAs was conducted between July 2018 and May 2019. Resource content was independently reviewed by two reviewers and scored for inclusion of each domain of CEnR core competencies. Domain scores across all resources and inter-rater reliability in scoring domains were assessed using descriptive statistics and Cohen's kappa coefficients. RESULTS/ANTICIPATED RESULTS: Overall, 214 resources available from 24 CTSAs were eligible for full review. Scoring discrepancies for at least one domain within a resource initially occurred in $51 \%$ of resources. "CEnR methods" (50.5\%; 108 of 214) and "Knowledge of/relationships with the community" (40.2\%; 86 of 214 ) were most frequently addressed and "Program evaluation" (12.1\%; 26 of 214) and "Dissemination and advocacy" (11.2\%; 24 of 214$)$ were least frequently addressed domains. Additionally, challenges were noted in navigating CTSA websites to access CEnR resources, and CEnR competency nomenclature was not standardized. DISCUSSION/SIGNIFICANCE OF IMPACT: Our findings guide CEnR stakeholders to identify CEnR resources accessible online and gaps to address in CEnR resource development. Standardized nomenclature for CEnR competencies is needed for effective CEnR resource classification. Uniform organization of CTSA websites may maximize navigability. CONFLICT OF INTEREST DESCRIPTION: In addition to the funding information listed previously (see above), within the last three years, R.J. Piasecki has been employed as: Project Coordinator, CEnR Online Learning Project, Johns Hopkins University School of Nursing (Current) Temporary Employee (Doctoral Student Intern), Michigan State University Institute for Health Policy (Current) Clinical RN, Intrastaff at the Johns Hopkins Health System (Past) Research Data Analysis Assistant, Maryland Institute for Emergency Medical Services (Past - contracted)

4463

\section{An Intervention Mapping Approach to Develop Interventions to Improve Access to Kidney Transplant}

Hannah D'Cunha ${ }^{1}$, Melissa Partin, Warren McKinney, Marilyn Bruin, and Allyson Hart ${ }^{1}$

${ }^{1}$ University of Minnesota CTSI

OBJECTIVES/GOALS: Kidney transplant is superior to dialysis for the treatment of end-stage kidney disease, but accessing transplant requires high patient engagement. We sought to develop a group counseling intervention with patients and their social support members using an evidence-based, stakeholder-engaged approach. METHODS/ STUDY POPULATION: We employed an Intervention Mapping approach to incorporate qualitative data from stakeholders on barriers to accessing kidney transplant. Data were collected from 13 focus groups of African American (AA) and white adult kidney transplant candidates and their social support networks in Minnesota and Georgia. We completed this process through (1) qualitative data collection, (2) utilizing data and intervention mapping methods to develop a conceptual framework to describe associations between behavioral determinants and desired outcomes, and (3) using these products to identify evidence-based approaches to modify behavioral determinants through a theory based intervention. RESULTS/ANTICIPATED RESULTS: Participants describe experiences of overwhelm, isolation, helplessness, and difficulty communicating. In addition, AA participants expressed distrust in the medical system. We systematically incorporated these themes into a conceptual model of behavior change that identifies determinants of necessary actions to obtain transplant, including knowledge, self-efficacy, reduced decisional conflict, and perception of social support. Evidence-based methods to modify these determinants, such as modeling, goal-setting, and mobilizing social support, were incorporated into the design of a group education and counseling intervention with an individualized risk calculator. 